NCU-HEP-k024

Mar 2006

rev. Nov 2006

ed. Feb 2007

\title{
Flavor Changing Higgs Decays in Supersymmetry with Minimal Flavor Violation
}

\author{
Abdesslam Arhrib ${ }^{1}$, Dilip K. Ghosh ${ }^{2}$, Otto \\ C. W. Kong ${ }^{3}$, and Rishikesh D. Vaidya ${ }^{4}$ \\ ${ }^{1}$ Département de Mathématiques, \\ Faculté des Sciences et Techniques, \\ B.P 416 Tanger, Morocco* \\ and Physics Division, \\ National Center for Theoretical Sciences, \\ P.O. Box 2-131 Hsinchu, Taiwan. \\ ${ }^{2}$ Department of Physics \& Astrophysics, \\ University of Delhi, Delhi 110007, India. \\ ${ }^{3}$ Department of Physics National Central University, \\ Chung-li, 32054, Taiwan. \\ ${ }^{4}$ Department of Theoretical Physics, \\ Tata Institute of Fundamental Research, \\ Mumbai 400 005, India.
}

We study the flavor changing neutral current decays of the MSSM Higgs bosons into strange and bottom quarks. We focus on a scenario of minimum flavor violation here, namely only that induced by the CKM matrix. Taking into account constraint from $b \rightarrow s \gamma, \delta \rho$ as well as experimental constraints on the MSSM spectrum, we

\footnotetext{
* Permanent Address.
} 
show that the branching ratio of $(\Phi \rightarrow b \bar{s})$ and $(\Phi \rightarrow \bar{b} s)$ combined, for $\Phi$ being either one of the CP even Higgs states, can reach the order $10^{-4}-10^{-3}$ for large $\tan \beta$, large $\mu$, and large $A_{t}$. The result illustrates the significance of minimal flavor violation scenario which can induce competitive branching fraction for flavor changing Higgs decays. This can be compared with the previous studies where similar branching fraction has been reported, but with additional sources of flavor violations in squark mass matrices. We also discuss some basic features of the flavor violating decays in the generic case.

PACS numbers: 
Supersymmetry (SUSY) is without doubt the most popular extension of the Standard Model (SM). Its possible discovery has been a major target of the Large Hadron Collider (LHC) program. Direct observation of some of the superparticles as well as indirect hints through SUSY enhancement of various rare processes in the SM are of great interest. Among the latter, processes characterized by flavor changing neutral currents (FCNCs) are particularly important. Especially within the framework of the minimal supersymmetric standard model (MSSM), the $b \rightarrow s \gamma$ transition is an important case example that has received the most attention [1, 2, 3]. Recently, there have been some studies on the related flavor changing Higgs decays (FCHDs) $\Phi \rightarrow b s$ (i.e. $\Phi \rightarrow b \bar{s}$ and $\Phi \rightarrow \bar{b} s$ combined; $\Phi$ in the case denotes $h^{0}, H^{0}$, or $\left.A^{0}\right)[4,5,6,47,8]$. The basic results indicate that good branching fractions for various Higgs states decaying into a $b$ and $s$ pair are admissible in some region of the parameter space surviving the experimental $B \rightarrow X_{s}+\gamma$ constraint. We consider careful, in depth, studies of the topic of great interest. In this brief letter, we report one step in the direction, pointing out that even for the case of a truly minimal flavor violation framework, substantial branching ratio is still admissible. By the minimal framework, we mean switching off all flavor off-diagonal squark mixings and use the CKM mixings as the sole source of flavor violation. The result is apparently outside the expectation of the previous authors. In fact, we find that the branching ratio can reach the $10^{-4}-10^{-3}$ level for large $\tan \beta$, large $\mu$, and large $A_{t}$, after the various relevant experimental constraints are put in. We will also briefly review what has been done in the generic case and discuss some related issues and suggestions for further studies.

Within the SM, fermions of the same charge have identical gauge interactions and there is only one Higgs doublet giving mass to all the fermions through various different Yukawa couplings. FCNC does not exist at tree level. Presence of more than one Higgs doublet can in general lead to FCNCs mediated by the physical Higgs states. Large FCNCs can be avoided in the so called "type-II" two Higgs doublet model, where one Higgs doublet $H_{u}$ gives mass to only the up-type quarks and the other Higgs doublet $H_{d}$ gives mass to only down-type quarks and charged leptons. Such a structure is protected against quantum corrections by a discrete symmetry under which the two Higgs doublets transform differently. The MSSM as a two Higgs doublet model has the type-II structure enforced, however, only through the holomorphy of the superpotential. This obviously cannot be effective once the soft SUSY breaking terms are taken into consideration. Flavor off-diagonal mass mixings 
among the squarks is a potentially very significant source of FCNC among quarks, starting at one loop level. Admitting R-parity violation of course introduces further sources of flavor violation [9]. We are, however, limiting ourselves to the case with a conserved $\mathrm{R}$ parity here. On the other hand, the nontrivial CKM matrix does give rise to FCNC processes through violation of the famous GIM cancellation among the charged current loops. This minimal, but unavoidable, flavor violation does induce extra SUSY contributions to FCNCs among quarks. In particular, it does contribute to $b \rightarrow s \gamma$ and $\Phi \rightarrow b s$.

Among the various FCNC processes, $b \rightarrow s \gamma$ is arguably the most extensively studied. In spite of the inherent complications from strong interactions, the inclusive $B \rightarrow X_{s}+\gamma$ result admits a relatively clean calculation that can be freed from scale and scheme uncertainties within the framework of RG-improved perturbation theory and heavy mass expansion based on the assumption of quark-hadron duality. The SM calculation has been completed to next to leading log precision[10] and the result stands in good agreement with the experimental numbers. Whereas, FCNCs in B decays have been extensively analyzed, not so much attention has been given to flavor changing interactions of Higgs boson. In fact, Higgs decays, being without complications that afflict B-decays, could provide fertile ground for explorations of FCNCs. This is especially the case for $\Phi \rightarrow b s$ in the large $\tan \beta$ domain. Such an investigation could also be interesting from the perspective of Higgs discovery. For example, it has been pointed out in Ref.[11] that the total number of FCNC heavy flavor events from supersymmetric Higgs boson interactions at the LHC can be as large as $\sim 10^{6}$. In the critical range for the lightest $\mathrm{CP}$ even Higgs mass $m_{h^{0}} \sim 90-130 \mathrm{GeV}$, the dominant decay mode $h^{0} \rightarrow b \bar{b}$ in large $\tan \beta$ region suffers huge QCD background. It even loses the race when compared to the cleaner signature of $h^{0} \rightarrow \gamma \gamma$, which has a much smaller width. The FCNC decay mode, being free from pure QCD background could possibly play a complementary role in identifying an MSSM CP even Higgs[12]. Another interesting property of the FCHDs is their non-decoupling nature in the limit of heavy SUSY spectrum limit[13]. In a pessimistic scenario where the superparticles are heavy enough to evade direct discovery at the next generation collider experiments, the non-decoupling behavior would then play a very important role in revealing SUSY.

A full diagrammatic calculation on the SM branching fraction for $h \rightarrow b s$ has been performed in Ref.[14]. The results are $10^{-7}\left(10^{-9}\right)$ for $m_{h}=100(200) \mathrm{GeV}$, in basic agreement with the earlier estimates based on dimensional analysis and power counting [6, 15]. 
Ref.[14] has also given a systematic analysis on the topic for the case of the two Higgs doublet model (without SUSY). For the type-I case, it has been found that the branching ratio falls in the range $10^{-5}-10^{-3}$ for small $\tan \beta \approx 0.1-0.5$; for the type-II model with the $b \rightarrow s \gamma$ constraint imposed, $10^{-5}-10^{-6}$ can be obtained. The CKM-induced, or SM, part of the results is dramatically larger than the up-sector counterpart, i.e. $\operatorname{Br}(h \rightarrow t c)$, of $10^{-13}[16]$. The down-sector case has two major advantages over that of the up-sector. Firstly, the large mass of the top inside the loop gives a bigger GIM violation. In addition, the $h \rightarrow b s$ channels are open even for $m_{h} \lesssim 2 M_{W}$ while the $h \rightarrow t c$ threshold of $m_{t}+m_{c}$ is about or above the Higgs to $W^{+} W^{-}$and $Z^{0} Z^{0}$ channels. While the channels with virtual W- or Z-boson(s) do contribute, for Higgs mass quite a bit below $2 M_{W}$, the FCHDs may enjoy a much better branching ratio. We take the opportunity to emphasize here that more favorable branching ratio for the FCHDs in general, and $\Phi \rightarrow b s$ in particular, are likely to be obtained where the widths of the usually dominating flavor conserving channels are suppressed. Below the $W^{+} W^{-}$threshold, $b \bar{b}$ is the dominating channel.

The MSSM is our focus model in this brief letter. For the present purpose, a good model background reference is provided by Ref.[17]. Our minimal flavor violation scenario means to focus on the absolutely unavoidable CKM-induced results. They are from the charged current loops involving the W-boson, the charged Higgs, or the charginos. Only the loop diagrams with the charginos are the truly SUSY contributions beyond what is available in the two Higgs doublet model. They include diagrams with a (Higgs to) chargino vertex or with a sfermion, most importantly the stop, vertex.

In the more general case, the soft SUSY breaking sector has many parameters admitting flavor violation. Taking MSSM as a low energy effective theory without assuming any particular model of SUSY breaking/mediation, the parameters would be simply generic phenomenological parameters. On the other hand, most of the available models of SUSY breaking/mediation assume a flavor-blind mechanism, reducing the source of flavor violation to a minimum. The latter is typically induced through the flavor non-universal Yukawa couplings and the RG-running due to the disparity between the involved scales. This has a preferential effectiveness on the terms involving the third generation and those with $L$ handed sfermions, say the so-called $L L$-mixings over $R R$-mixings. The $L R$-mixings introduced through the trilinear $A$-terms may also have important flavor off-diagonal results. In fact, the $A$-terms themselves are direct Higgs-sfermion couplings. Such flavor off-diagonal 
couplings of course induce FCHDs, especially through a gluino diagram with the strong QCD coupling vertices.

Flavor violation in the soft SUSY breaking sector adds to the CKM-induced effect of the charged current loops. In addition, there are new contributions from the gluino and neutralino loops. Again, the former is particularly important due to the strong QCD coupling. Hence, with significant soft sector flavor violation, the gluino-sfermion loops will dominate the FCHDs, while for small or vanishing soft sector flavor violation, the chargino-sfermion loops should be more important. We would also like to point out that the $L L$-mixings among the squarks of the up- and down-sector originate from the same squark doublet mass terms. They are not identical, but related through a unitary rotation by the CKM matrix. Hence, only with a doublet squark $\widetilde{Q}$ soft mass matrix proportional to the identity matrix could the $L L$-mixings be completely removed. We stick to this true minimal flavor violation case for our numerical study here.

A real Higgs particle still awaits discovery while a $b$ - $s$ type flavor violation beyond what is predicted within the SM is already stringently constrained, especially in the very good agreement of the SM $B \rightarrow X_{s}+\gamma$ number with the experimental results - the world average is given by [18]

$$
\operatorname{Br}\left[B \rightarrow X_{s}+\gamma\left(E_{\gamma}>1.6 \mathrm{GeV}\right)\right]=(3.34 \pm 0.38) \times 10^{-4}
$$

while the next to leading log theoretical number for the SM [10]

$$
\operatorname{Br}\left[B \rightarrow X_{s}+\gamma\left(E_{\gamma}>1.6 \mathrm{GeV}\right)\right]_{\mathrm{SM}}=(3.57 \pm 0.30) \times 10^{-4}
$$

agreeing within $1 \sigma$. Obviously, the only relevant theoretical predictions for a FCHD $\Phi \rightarrow b s$ from any model have to be restricted to parameter space regions examined to survive the constraint from the radiative $B$ decay.

For the MSSM, the partonic level $b \rightarrow s \gamma$ transition has the dominant contributions to the Wilson coefficients of the magnetic and chromomagnetic penguin operators. They are induced by the charged current loops with the W-boson, charged Higgs, and charginos, as well as the gluino and neutralino loops. CKM-induced flavor violation contributes only to the charged current loops, which are hence the only directly relevant part here. Note that the charged Higgs contribution is of the same sign as that of W-boson, whereas the sign of chargino contributions depend on the sign of the product $\mu A_{t}$. In the interesting 
limit of large $\tan \beta$, sign of the large chargino contributions completely dictate the resulting branching fraction. The chargino contributions escape the GIM cancellation through the higgsino components, with $\tan \beta$ enhanced Yukawa couplings. It becomes highly preferable to maintain a relative negative sign between the $\mu$ and the $A_{t}$ parameters in order to bring about cancellations between positive contributions from W-boson and charged Higgs and negative contribution of chargino loops. That allows the branching fraction of the radiative decay to fall within the experimentally allowed range while keeping the Higgs spectrum light. The FCHD $\Phi \rightarrow b s$ for MSSM (with conserved R parity) has been studied in Refs. [4, 5, 6, 8] (see also Refs [7, 11]). Refs. [4, 6] consider only the gluino loop contribution. Ref. [4] claims branching ratio of the order of few percents, but it has not put in the $b \rightarrow s \gamma$ constraint. The latter implementation has been performed in Ref. [6], with realistic branching fraction in the range of $10^{-4}-10^{-3}$ obtained. Much bigger numbers are shown to be possible but considered unnatural as cancellation among the SM contribution and the extra, gluino, contribution to $b \rightarrow s \gamma$ is required. In our opinion, the part of the results is of limited interest not so much because it is "unnatural" as it is unrealistic. After all, the interesting scenarios of the flavor violation typically involve cancellations among and special suppression of some ingredients. For instance, we do want cancellations among the various MSSM contributions to $b \rightarrow s \gamma$, and large $\Phi \rightarrow b s$ is much related to where the dominating $b \bar{b}$ channel is suppressed. The latter may be considered a cancellation effect among the various contributions to the effective coupling. As Ref.[6] does not include SUSY electroweak contributions, the results relying on a cancellation of the gluino part against the others, hence, have no realistic value for the full MSSM. As mentioned above, with flavor off-diagonal soft masses small enough to remove the dominance of the gluino contribution, the chargino one with the non-negotiable CKM-induced part becomes very significant. The $10^{-4}-10^{-3}$ numbers for the branching fraction results, however, are already quite encouraging. More detailed careful studies are called for.

In contrast to Refs. [4, 6], Ref. [5] concentrates on the contributions from the electroweak part of the model, obtaining branching fraction in the range of $10^{-5}-10^{-3}$. Their implementation of the $b \rightarrow s \gamma$ constraint with the help of micrOMEGAs[19], however, leaves much room for improvement as the code is based on a minimal flavor violation scenario as described in Ref.[2]. A more consistent implementation has been performed in Ref.[8]. All the published studies other than Ref.[8] take as the source of flavor violation the $L L$-mixings 
in squark masses of the second and third generations. While for most of the models on SUSY breaking/mediation the $L L$-mixings is commonly expected to be larger than $L R$ - or $R R$ mixings, purely phenomenological results on the latter, independent of the SUSY breaking models, are of interest in their own right. Moreover, the argument that $L R$-mixings are of less interest because they are more stringently constrained by $b \rightarrow s \gamma$ does not stand. The $L R$-mixings are more constrained because they contribute more effectively to $b \rightarrow s \gamma$, which requires a chiral flip. Likewise, they contribute more effectively to $\Phi \rightarrow b s$. As mentioned above, the $L R$-mixings means flavor off-diagonal $A$-terms and hence Higgs-sfermion vertices. As it is the admissible $\Phi \rightarrow b s$ surviving the $b \rightarrow s \gamma$ constraint that we are after, the $L R$ mixings, or rather flavor off-diagonal $A$-terms, do not clearly appear to be at a position of disadvantage when compared to the $L L$-mixings soft mass terms. This is especially relevant with the gluino contribution.

It is interesting to see different parts of the contributions to $\Phi \rightarrow b s$ within MSSM. However, having the electroweak contribution without the gluino one is not realistic. The more interesting question there is if the electroweak contribution could dominate over the gluino one. The limiting case of that is the minimal CKM-induced flavor violation scenario on which we will report explicit results below. More detailed comprehensive studies for the generic case is certainly in order. Ref.[8] is an attempt in the direction. However, the treatment of the up- and down-sector $L L$-mixings as independent or identical parameters is not exactly correct. All discussions about the electroweak contributions have not separated the CKM-induced part from that which has the soft SUSY parameters as its source. We take up the task here, as a step to reveal more interesting details of the FCHDs. Our result here that even the purely CKM-induced contribution can get to the $10^{-4}-10^{-3}$ level would likely be beyond expectations.

For all the numerical results reported below, we switch off all soft sector flavor violations. The structure of the MSSM results is well known and the analytical formulas involved in the calculation are by now standard. We have no disagreement with previous authors on the matter. Hence, unless where necessary for the illustration of the issues under discussion, we will mostly refrain from showing such expressions in this brief letter but only highlight the more important points involved and give relevant references. Note that we stick to full mass eigenstate formalism, no mass-insertion approximation applied.

We have used for the $B \rightarrow X_{s}+\gamma$ calculation the NLO expressions given in Ref.[20], 
while for the Wilson coefficients $C_{7}$ and $C_{8}$ we have used the expression given in Ref.[21] at $\mathrm{LO}$ in the framework of MSSM with CKM as the only source of flavor violation. We impose the constraint asking the theoretical number to lie within the $3 \sigma$ experimental bounds.

We have computed Higgs decay width for $\Gamma_{\Phi}$ for all the three mass eigenstates $h^{0}, H^{0}$, or $A^{0}$, including the following partial widths at the leading order :

$$
\begin{aligned}
\Gamma_{\Phi}= & \sum_{f} \Gamma(\Phi \rightarrow f \bar{f})+\Gamma(\Phi \rightarrow V V) \\
& +\Gamma\left(\Phi \rightarrow V H_{i}\right)+\Gamma\left(\Phi \rightarrow H_{i} H_{j}\right)+\Gamma(\Phi \rightarrow \text { SUSY particles })
\end{aligned}
$$

with expressions in accordance with Ref.[17]. QCD corrections to $\Phi \rightarrow f \bar{f}$ and $\Phi \rightarrow$ $\left\{V^{*} V^{*}, V V^{*}, V^{*} H_{i}\right\}$ decays are not included in the width. Since the width of $\Phi \rightarrow b s$ may become comparable to $\Phi \rightarrow\{g g, \gamma \gamma\}$, it is necessary to include $\Phi \rightarrow\{g g, \gamma \gamma\}$ in the computation as we have done. All the computations of the rate for FCHD are done with the help of the packages FeynArts,FormCalc [22], and with LoopTools and FF for numerical evaluations [23, 24]. The one-loop amplitudes are evaluated in the 't Hooft-Feynman gauge using dimensional regularization.

The MSSM Higgs sector is parametrized by the mass of the CP-odd $M_{A}$ and $\tan \beta$ while the top quark mass and the associated squark masses enter through radiative corrections [25]. In our study we include the leading corrections only, with the Higgs masses given by

$$
\begin{aligned}
& m_{h^{0}, H^{0}}^{2}=\frac{1}{2}\left[M_{A Z}^{2} \mp \sqrt{M_{A Z}^{4}-4 M_{A}^{2} M_{Z}^{2} \cos ^{2} 2 \beta-4 \epsilon M_{A}^{2} \sin ^{2} \beta+M_{Z}^{2} \cos ^{2} \beta}\right] \\
& \tan 2 \alpha=\frac{M_{A}^{2}+M_{Z}^{2}}{M_{A}^{2}-M_{Z}^{2}+\epsilon / \cos 2 \beta} \tan 2 \beta, \quad-\frac{\pi}{2} \leq \alpha \leq 0,
\end{aligned}
$$

where

$$
M_{A Z}^{2}=M_{A}^{2}+M_{Z}^{2}+\epsilon, \quad \epsilon=\frac{3 G_{F}}{\sqrt{2} \pi^{2}} \frac{m_{t}^{4}}{\sin ^{2} \beta} \log \left[\frac{m_{\tilde{t}_{1}} m_{\tilde{t}_{2}}}{m_{t}^{2}}\right] .
$$

Our major result is illustrated in Fig, 1, in which we plot the branching fraction of $h^{0} \rightarrow b s$ in the plane $\left(M_{A}, \tan \beta\right)$ (left panel) and the decay width in $\mathrm{GeV}$ as a function of $M_{A}$ (right panel) for $M_{\mathrm{SUSY}}=800 \mathrm{GeV}, \mu=1 \mathrm{TeV}$, and a common value for the $A$-terms $\left(A_{t}=\right.$ $\left.A_{b}=A_{\tau}\right)$ at $-1.6 \mathrm{TeV}\left(=-\operatorname{sign}(\mu) 2 M_{\mathrm{SUSY}}\right)$, and $M_{2}=200 \mathrm{GeV} ; M_{\mathrm{SUSY}}$ is the common soft mass for all squarks involved. Note that we take $A_{t}$ with a relative negative sign to the $\mu$-parameter as preferred for $b \rightarrow s \gamma$. As also noted in Ref.[6], large branching fraction for $h^{0} \rightarrow b s$ is obtained in the parameter range of the small $\alpha_{\text {eff }}$ scenario[26]. As $\tan \beta$ runs 

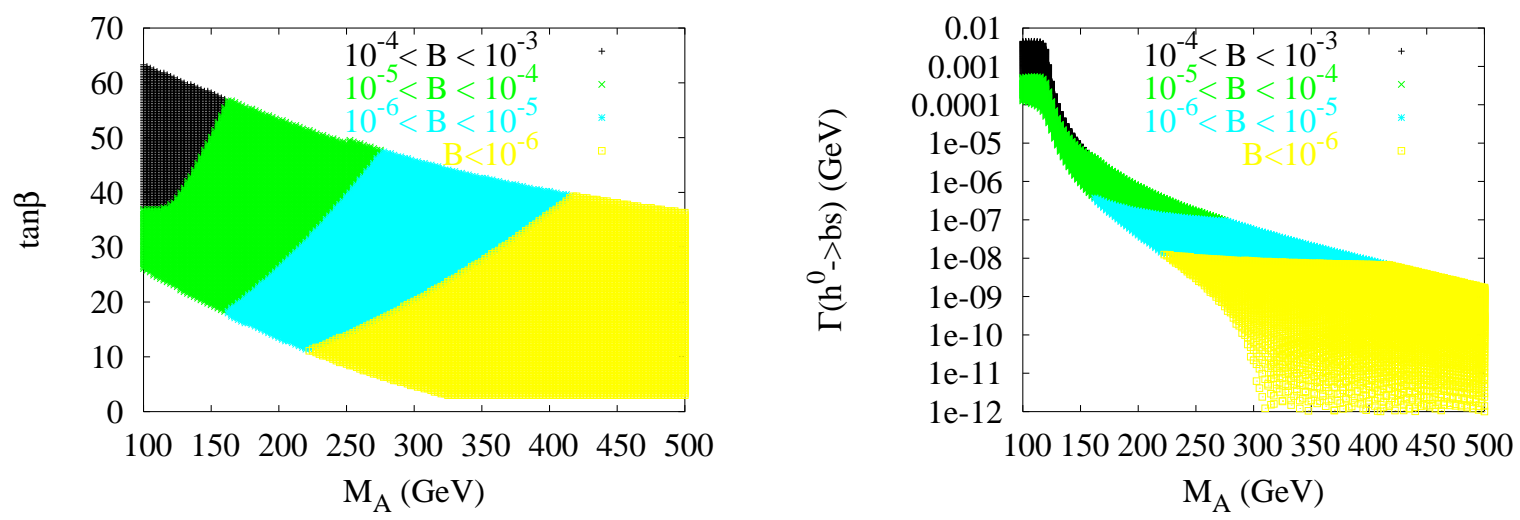

FIG. 1: Branching ratio of $h^{0} \rightarrow b s$ in the plane of $M_{A}, \tan \beta$ (left panel) and the decay width $\Gamma\left(h^{0} \rightarrow b s\right)$ in $\mathrm{GeV}$ (right panel) as a function of $M_{A}$ with $2<\tan \beta<65$, for $M_{\text {SUSY }}=800 \mathrm{GeV}$, $\mu=1 \mathrm{TeV}, A_{t}=A_{b}=A_{\tau}=-1.6 \mathrm{TeV}$, and $M_{2}=200 \mathrm{GeV}$. From up-left to down-right $B=$ $B\left(h^{0} \rightarrow\right.$ bs $)$ is: $10^{-4}<B<10^{-3}, 10^{-5}<B<10^{-4}, 10^{-6}<B<10^{-5}, B<10^{-6}$.
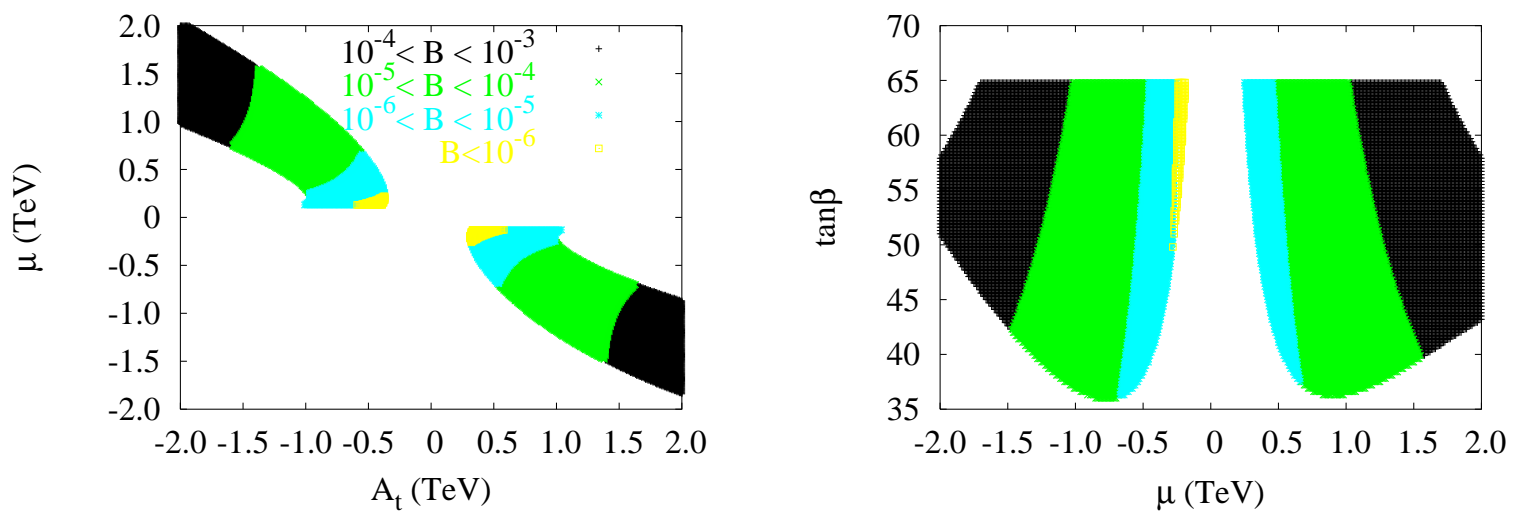

FIG. 2: Branching ratio of $h^{0} \rightarrow b s$ in the $\left(A_{t}, \mu\right)$ plane with $\tan \beta=45$ (left panel) and $(\mu, \tan \beta)$ with $A_{t}=-\mu$ (right panel) with $M_{A}=130 \mathrm{GeV}, M_{\mathrm{SUSY}}=800 \mathrm{GeV}$, and $M_{2}=200 \mathrm{GeV}$. From left to right or right to left: $10^{-4}<B<10^{-3}, 10^{-5}<B<10^{-4}, 10^{-6}<B<10^{-5}, B<10^{-6}$.

here, it tunes the loop corrections to the Higgs mass, i.e. it tunes $\alpha_{\text {eff }}$. Small $M_{A}$ and large $\tan \beta$, and positive $\mu$ are preferred. As one can see from left plot, large branching fraction $\mathcal{B}>10^{-5}$ is obtained for large $\tan \beta \gtrsim 30$ and $M_{A}<250 \mathrm{GeV}$. The unmarked regions in the plots are excluded by the violations of either $b \rightarrow s \gamma, \delta \rho$, or one of the experimental limit on neutralino and chargino masses.

In Fig 2, we show the branching fraction of $h^{0} \rightarrow$ bs in the plane of $\left(A_{t}, \mu\right)$ with $\tan \beta=45$ 

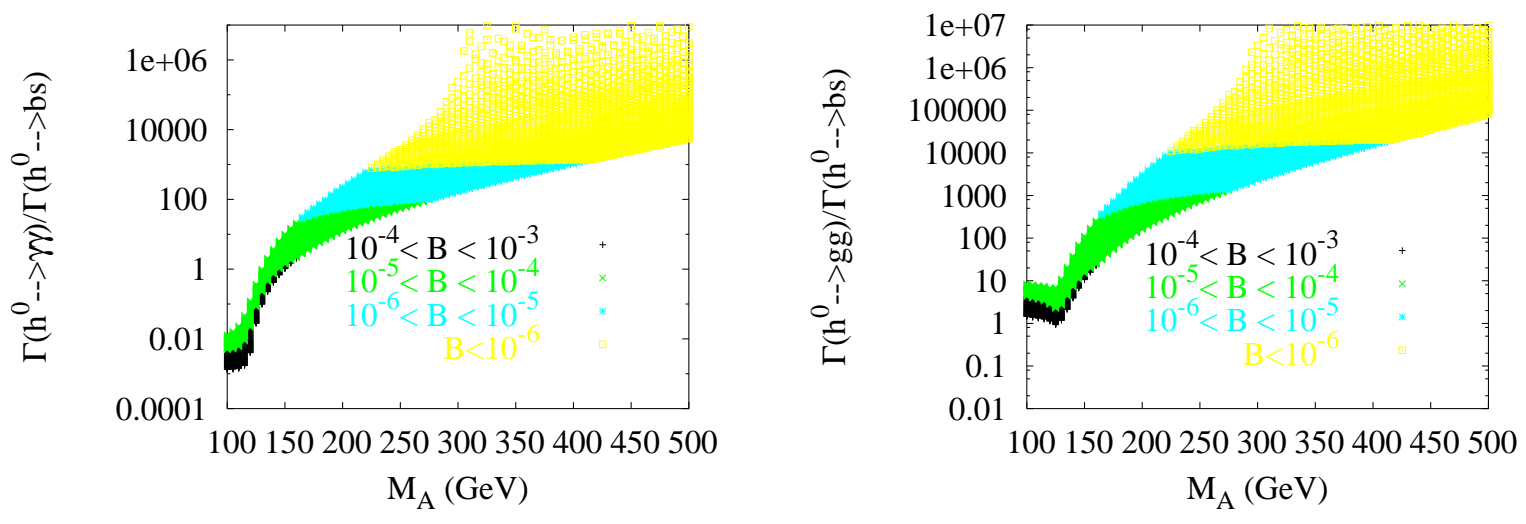

FIG. 3: Ratio of $\Gamma\left(h^{0} \rightarrow \gamma \gamma\right) / \Gamma\left(h^{0} \rightarrow b s\right)$ (left panel) and $\Gamma\left(h^{0} \rightarrow g g\right) / \Gamma\left(h^{0} \rightarrow b s\right)$ (right panel) as a function of $M_{A}$ with $2<\tan \beta<65$. The other parameters same as for Fig!1. From down-left to up-right $B=B\left(h^{0} \rightarrow b s\right)$ is: $10^{-4}<B<10^{-3}, 10^{-5}<B<10^{-4}, 10^{-6}<B<10^{-5}, B<10^{-6}$.

(left panel) and $(\mu, \tan \beta)$ with $A_{t}=-\mu$ (right panel), respectively, for $M_{A}=130 \mathrm{GeV}$, $M_{\mathrm{SUSY}}=800 \mathrm{GeV}, M_{2}=200 \mathrm{GeV}$. It is clear that large branching fraction is obtained for large $A_{t}$, large $\mu$ as well as large $\tan \beta$. Similar to Fig 1, unmarked regions in the plots are excluded by the violations of either $b \rightarrow s \gamma, \delta \rho$, or one of the experimental limit on neutralino and chargino masses. We mention in passing that from the left plot one can see that $b \rightarrow s \gamma$ favors $\mu A_{t}$ to be negative.

We illustrate in Fig 3 , the ratios $\Gamma\left(h^{0} \rightarrow \gamma \gamma\right) /\left(\Gamma\left(h^{0} \rightarrow b s\right)\right)$ (left panel) and $\Gamma\left(h^{0} \rightarrow\right.$ $g g) /\left(\Gamma\left(h^{0} \rightarrow s b\right)\right)$ (right panel) as a function of $M_{A}$ for $2<\tan \beta<65$, and otherwise the same model parameters as in Fig:1. From the left plot one can read that $\Gamma\left(h^{0} \rightarrow b s\right)$ can be both larger or smaller than $\Gamma\left(h^{0} \rightarrow \gamma \gamma\right)$ while from the right plot we can see that $\Gamma\left(h^{0} \rightarrow g g\right)$ is almost all the time larger than $\Gamma\left(h^{0} \rightarrow b s\right)$. The $h^{0} \rightarrow b s$ partial width is in fact comparable to that of $h^{0} \rightarrow \gamma \gamma$, as we illustrated in Fig, 3 .

Situation for the heavier CP even state $H^{0}$ is similar, as illustrated in Fig, 4 , based on the same set of parameter input. As in the case of $h^{0}$, the branching fraction of $H^{0} \rightarrow b s$ can reach $10^{-4}$ level. The corresponding result for the pseudoscalar is very similar to $H^{0}$ and we do not show it here.

At this point, some comments on the issues related to the limitations of the approximation used in our calculations are in order. It has been well appreciated that there are important QCD corrections to the Higgs to fermion decay widths as well as significant (resummed) 

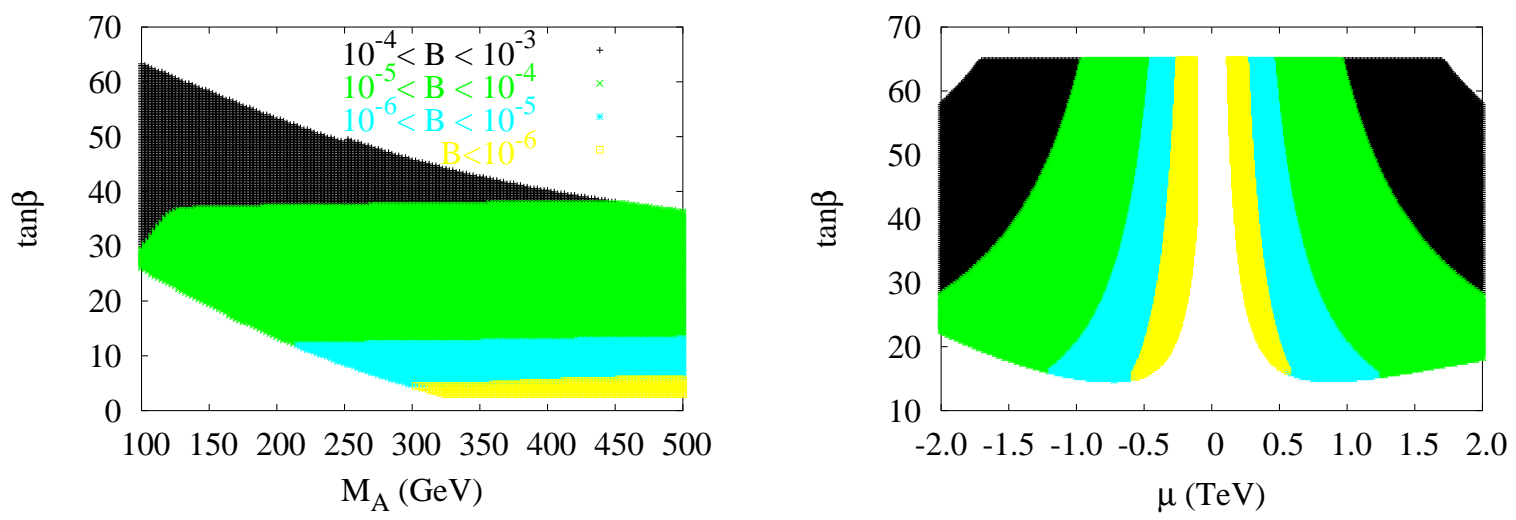

FIG. 4: Branching ratio of $H^{0} \rightarrow b s$ in the $M_{A}, \tan \beta$ plane (left panel) and $(\mu, \tan \beta)$ plane (right panel), the former with $\mu=1 \mathrm{Tev}$, the latter with $M_{A}=250 \mathrm{GeV}, A_{t}=-\mu$. The other unspecified parameters are same as for Fig[1. Left panel: from up-left to down-right $B=B\left(H^{0} \rightarrow b s\right)$ is: $10^{-4}<B<10^{-3}, 10^{-5}<B<10^{-4}, 10^{-6}<B<10^{-5}, B<10^{-6}$. Right panel: From left to right or right to left: $10^{-4}<B<10^{-3}, 10^{-5}<B<10^{-4}, 10^{-6}<B<10^{-5}, B<10^{-6}$.

large $\tan \beta$ corrections. We have not implemented the corrections into our calculations here, for the main reason that a consistent implementation is very involved, in fact quite beyond the scope of present work or what has been done by other authors on this topic so far. Moreover, we believe the present analysis does illustrate at least qualitatively some interesting physics which is not going to be totally invalidated by a complete consistent higher order calculations incorporating the corrections. While how to implement the above corrections to the, flavor-conserving Higgs decays is well known and even built into the standard numerical codes, a consistent implementation here requires the simultaneous implementation onto the flavor changing $\Phi \rightarrow b s$ part as well as the $b \rightarrow s \gamma$ background constraint. A partial implementation, say onto flavor-conserving Higgs decays only, will likely give misleading results - a point also made in Ref.[6]. For example, QCD correction is known to reduce the Higgs to $b \bar{b}$ width. However, it will more or less have a similar effect on the $\Phi \rightarrow b s$ width. In fact, one may expect some partial cancellation between the two rendering the overall effect on the branching ratio we are interested in less than what the correction to Higgs to $b \bar{b}$ in itself may indicate. And there is still the corresponding corrections to $b \rightarrow s \gamma$ to worry about. The situation for the large $\tan \beta$ corrections is similar. Of course part of the corrections involved may be somewhat sensitive to the values of the SUSY parameters that 
have otherwise minimal role to play here - the gluino mass is one clear example. Thus, to the leading order, our results are consistent and not inferior to the results reported in the previous works. Rather the effect of CKM induced contributions have been brought out more clearly.

We expect our conclusion to qualitatively remain valid over quite some region of the general SUSY parameter space. Let us take a look at the plausible numerical impact of the corrections here, focusing on the small Higgs mass region where they have a strong effect. The region is also where our results look most interesting. QCD correction reduces the Higgs to $b \bar{b}$ (and other fermion channels) width. Our focus here being the branching ratio of the flavor changing decays, within the constraint of flavor violation admissible in $b \rightarrow s \gamma$. Had we naively included the QCD corrections to the Higgs to $b \bar{b}$, we may have ended up in false enhancement. The (resummed) large $\tan \beta$ correction is more relevant here, as our results give interesting branching ratio mostly in the large $\tan \beta$ region. The $\tan \beta$ effect is, in first order, a correction on the $b$-Yukawa coupling. Depending on the SUSY parameters, the effect can reach a $60-70 \%$ enhancement of the (like $b \bar{b}$ ) width. However, a substantially reduced effect due to partial cancellation of various contributions is not inconceivable. As the major contribution to the $\Phi \rightarrow b s$ channel discussed here comes from the chargino-stop diagram, the large $\tan \beta$ correction to the flavor changing width would be of higher order. However, there is still the effect on the $b \rightarrow s \gamma$ constraint to be considered. On the whole, we think it is reasonable to expect that the correction would not change the admissible branching ratio by an order of magnitude.

We further illustrate some naive results on the $h^{0} \rightarrow b s$ branching ratios with flavor violating soft mass inputs to give the readers a direct comparison. In Fig 5 , the branching ratios for the, separate, cases with nonzero $L L-, R R$-, or $R L$ - (flavor off-diagonal $A$-term) mixings among the second and third family squarks are illustrated, for otherwise the same model parameters. Two specific values of $\tan \beta, 15$ and 45, are chosen for clear illustration. Notice that the minima of the curves apart from the $L L$ cases are at zero mixings, with value given by the independent CKM induced contribution. For the cases of the $L L$-mixings, The CKM induced effect cannot be separated as an independent part. In fact, the minima are off-set from zero mixings and go deeper as the CKM induced effect is partially canceled. The pairs of curve for the two $\tan \beta$ values also illustrate the basic trend of dependence on the parameter. The $L L$ and $R R$ cases have the $\tan \beta$ enhancement as for the CKM only results. 

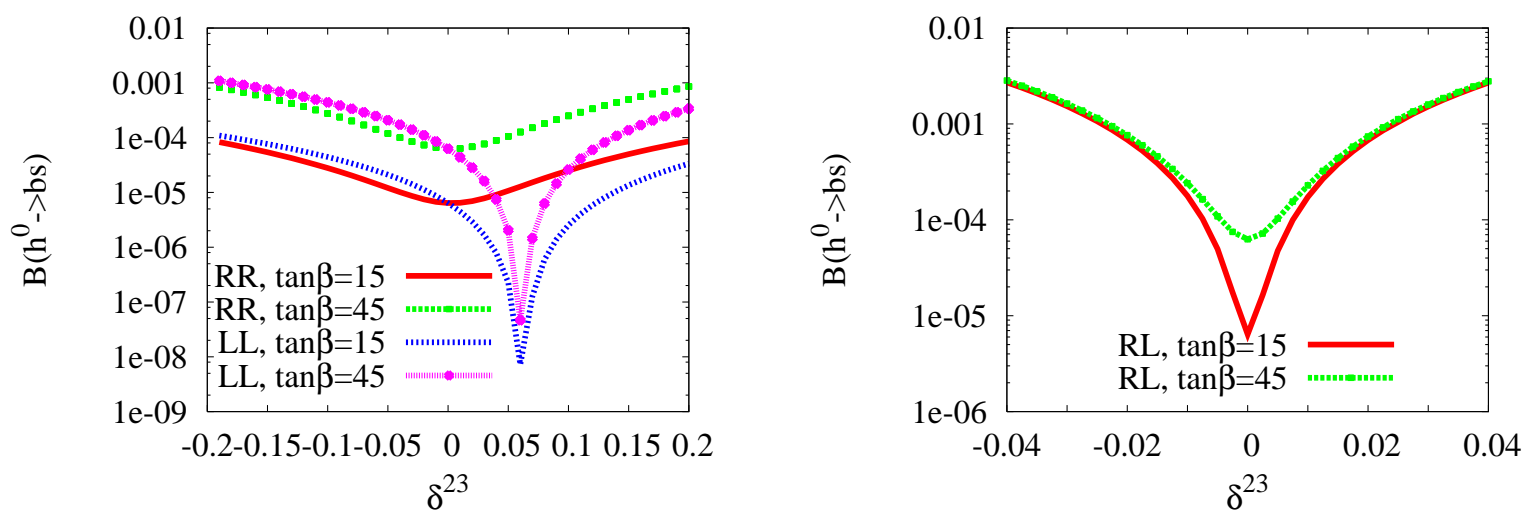

FIG. 5: Naive $\left(h^{0} \rightarrow b s\right)$ branching ratio results with flavor violating soft mass inputs, for comparison. The other parameters same as for Fig.1.

For the $R L$ case, however, as it is actually a linear combination of the soft $(A-)$ term and the $\tan \beta$-dependent $F$-term that matters, $\tan \beta$-dependence fades out at large $(A$-term) mixings. The plots are shown only for the purpose of illustrating the basic theoretical feature. Hence, no checking against $b \rightarrow s \gamma$ or other constraints applied.

In summary, we have discussed the potentially experimentally interesting flavor changing Higgs decays $\Phi \rightarrow b s$ for the MSSM, and presented explicit numerical results focusing on the minimal flavor violation scenario. The scenario has FCNC induced through mostly chargino loops with the CKM mixing as the sole, and unavoidable, source of flavor violation. We illustrate that the branching fractions for the decays of all three Higgs states, after factoring in the other major experimental constraints such as $b \rightarrow s \gamma$, can still reach the range of $10^{-4}-10^{-3}$, without any extra flavor violation of SUSY origin. The numbers are comparable with that obtained by the other authors through assuming flavor violating soft squark masses. Our calculations have not incorporated the major QCD and large $\tan \beta$ corrections, the consistent implementation of which still has to be performed. We explained the related concerns and our belief that the interesting branching ratio illustrated is very unlikely to be altered by an order of magnitude.

Acknowledgements: We would like to thank F. Borzumati, Y. Hsiung and G. Mazumdar for helpful discussions. O.K. is partially supported by research grants number 94-2112-M- 
008-009 and 95-2112-M-008-001 from the NSC of Taiwan.

[1] For the MSSM studies of $b \rightarrow s \gamma$ please see S. Bartolini et al., Nucl. Phys. B353 (1991), 591; N. Oshimo, Nucl. Phys. B404 (1993), 20; F. Borzumati, Z. Phys. C 63(1994), 291.

[2] G. Degrassi et al., J. High Energy Phys. 0012 (2000), 009. For further details and references, see the review by T. Hurth, Rev. Mod. Phys. 75 (2003), 1159.

[3] For a comprehensive analysis of $b \rightarrow s \gamma$ in the R-parity violating framework, see O.C.W. Kong and R. Vaidya, Phys. Rev. D71 (2005), 055003; Phys. Rev. D72 (2005), 014008 and references therein.

[4] A. M. Curiel, M. J. Herrero, and D. Temes, Phys. Rev. D 67 (2003), 075008.

[5] A. M. Curiel, M. J. Herrero, W. Hollik, F. Merz, and S. Penaranda, Phys. Rev. D 69 (2004), 075009 .

[6] S. Bejar, F. Dilme, J. Guasch, and J. Sola, JHEP 0408 (2004), 018.

[7] W. Hollik, S. Peñaranda, and M. Vogt, arXiv:hep-ph/0511021.

[8] T. Hahn, W. Hollik, J.I. Illana, and S. Peñaranda, arXiv:hep-ph/0512315.

[9] See, for example, O.C.W. Kong, Int. J. Mod. Phys. A 19 (2004), 1863 for a clear elaboration of the basic flavor structure.

[10] For studies within SM see, P. Gambino and M. Misiak, Nucl. Phys. B 611 (2001), 338. Also see A. Kagan and M. Neubert, Eur. Phys. J C 7 (1999), 5. A complete NLL calculation is given in Buras et al., Nucl. Phys. B 631 (2002), 219. A recalculation under the framework of soft collinear effective theory is given by M. Neubert, Eur. Phys. J. C40 (2005), 165.

[11] S. Bejar, J. Guasch, and J. Sola, JHEP 0510 (2005), 113.

[12] The problem here is with $s$-jet identification, as versus $b$ - as well as $u$ - and $d$-jets, which is very difficult but not impossible. - Y. Hsiung, private communiation; G. Mazumdar, private communiation.

[13] See, for example, Refs. [5, 7] and references therein for more discussions on the non-decoupling aspects.

[14] A. Arhrib, Phys. Lett. B 612 (2005), 263.

[15] S. Bejar, J. Guasch, and J. Sola, Nucl. Phys. B 675 (2003), 270.

[16] G. Eilam, J. L. Hewett, and A. Soni, Phys. Rev. D 44 (1991), 1473 [Erratum, ibid. D 59 
(1999), 039901]; B. Mele, S. Petrarca and A. Soddu, Phys. Lett. B 435 (1998), 401.

[17] A. Djouadi, arXiv:hep-ph/0503173.

[18] S. Eidelman et al. [Particle Data Group], Phys. Lett. B 592 (2004) 1; and 2005 update at http://pdg.lbl.gov/

[19] G. Belanger, F. Boudjema, A. Pukhov, and A. Semenov, Comput. Phys. Commun. 149 (2002), 103.

[20] A. L. Kagan and M. Neubert, Phys. Rev. D 58(1998), 094012.

[21] M. Aoki, G. C. Cho, and N. Oshimo, Nucl. Phys. B 554 (1999), 50.

[22] T. Hahn, Comput. Phys. Commun. 140 (2001), 418; T. Hahn, C. Schappacher, Comput. Phys. Commun. 143 (2002), 54; T. Hahn, M. Perez-Victoria, Comput. Phys. Commun. 118 (1999), 153; T. Hahn, Nucl. Phys. Proc. Suppl. 135 (2004), 333; J. Küblbeck, M. Böhm, A. Denner, Comput. Phys. Commun. 60 (1990), 165; see also T. Hahn: http://www.feynarts.de

[23] G.J. van Oldenborgh, Comput. Phys. Commun. 66 (1991), 1.

[24] T. Hahn, Acta Phys. Polon. B 30 (1999), 3469; T. Hahn and M. Rauch, arXiv:hep-ph/0601248.

[25] J. F. Gunion and A. Turski, Phys. Rev. D 39 (1989), 2701 M. S. Berger, Phys. Rev. D 41 (1990), 225; Y. Okada, M. Yamaguchi and T. Yanagida, Prog. Theor. Phys. 85 (1991), 1.

[26] M. Carena, S. Heinemeyer, C.E.M. Wagner, and G. Weiglein, Eur. J. Phys. C 26 (2003), 601; M. Carena, S. Mrenna, and C.E.M. Wagner, Phys. Rev. D 62,(2000), 055008. 